This column features important events and notes of interest occurring at the Canadian Public Health Association National Office.

Cette rubrique porte sur les événements importants et publie des notes d'information sur ce qui se passe au siège social de l'Association canadienne de santé publique.

\section{CPHA and the global effort to strengthen responses to HIV/AIDS}

I was privileged to participate in a news conference on 1 December 1999, World AIDS Day, where Canada's International Cooperation Minister, Maria Minna, announced $\$ 50$ million in new funding to support projects that are successfully fighting HIV/AIDS in Africa. CPHA's Southern African AIDS Training (SAT) Programme is one of three projects that will have access to this new funding to negotiate new and expanded work in prevention, education and training.

The SAT Programme, funded by the Canadian International Development Agency (CIDA) since 1990, provides technical and financial support to local NGOs and community-based organizations in their efforts to put into place effective communitybased prevention and support responses to the HIV/AIDS pandemic in eleven southern African countries. Based in Harare, Zimbabwe, SAT concentrates on the poorest $20 \%$ of the population, reaching communities in southern Africa with the least access to services and support. This region of 165 million people currently has some 4-6 million HIV-infected cases recorded, and anticipates more than 8 million cases by the year 2010 .

Active in both urban and rural settings, the SAT Programme currently supports over 120 community initiatives throughout southern Africa.

SAT established a regional training initiative entitled School Without Walls (SWW) in partnership with a number of local experienced community organizations and NGOs willing to share their institutional and AIDS expertise with others. SWW aims to accelerate learning and programme development in communities which are struggling within the context of the ever-changing AIDS epidemic, and emphasizes a non-formal, learning by seeing and doing approach in structured field situations.

The SAT Programme is strengthening its gender training and human rights analysis activities to provide partners with better support. By giving careful and culturally sensitive consideration to gender relations, human rights and development issues, SAT aims to open new and improved avenues for HIV/AIDS prevention, research and practice.

For more information on the SAT Programme, contact Brenda Millar, Programme Officer, Tel: 613-725-3769, ext 174, e-mail: bmillar@cpha.ca

Gerald H. Dafoe

Chief Executive Officer

\section{L'ACSP et les efforts mondiaux pour renforcer les mesures dans la lutte contre le VIH/sida}

J'ai eu le privilège d'assister à une conférence de presse le $1^{\mathrm{er}}$ décembre dernier, Journée mondiale du SIDA, au cours de laquelle Maria Minna, ministre canadienne de la Coopération internationale, a annoncé une nouvelle contribution de 50 millions de dollars pour appuyer la prolongation de projets qui luttent efficacement contre le VIH-sida en Afrique. Le Programme d'Afrique australe de formation sur le sida (SAT), que gère l'ACSP, est l'un des trois programmes qui bénéficieront de ces fonds supplémentaires pour négocier de nouvelles activités ou en élargir d'autres dans les domaines de la prévention, de l'éducation et de la formation.

Le Programme SAT, que finance l'Agence canadienne de développement international (ACDI) depuis 1990, apporte une assistance technique et financière aux ONG locales et aux organismes communautaires pour les aider à mettre en place des mesures communautaires efficaces de soutien et de prévention face à la pandémie du VIH/sida qui sévit dans onze pays d'Afrique australe. Le Programme SAT, basé à Harare au Zimbabwe, fait porter l'essentiel de ses efforts sur les 20 p. 100 d'individus les plus pauvres de la population, en aidant les communautés d'Afrique australe qui ont le moins accès aux services et qui reçoivent peu de soutien. Cette région qui compte 165 millions de personnes, enregistre à l'heure actuelle de 4 à 6 millions de cas déclarés de séropositivité au VIH, et s'attend à ce que ce chiffre passe à 8 millions de cas d'ici 2010 .

Présent à la fois en milieu urbain et rural, le Programme SAT a financé plusieurs centaines d'initiatives dans toute l'Afrique australe.

Le Programme SAT a lancé une initiative régionale de formation, intitulée L'école hors les murs, en partenariat avec plusieurs organismes communautaires expérimentés, et avec des ONG disposées à partager leur connaissance des institutions et du sida. L'école hors les murs a pour objectif d'accélérer l'apprentissage et l'élaboration de programmes dans les communautés devant composer avec l'évolution constante de l'épidémie du sida, et insiste sur un apprentissage sur le tas, consistant à voir et à faire en situation, sur le terrain.

Le Programme SAT renforce ses activités de formation aux différences hommes-femmes et d'analyse des droits humains afin de mieux épauler ses partenaires. En faisant preuve de sensibilité culturelle dans sa façon de traiter des questions relatives aux différences entre les hommes et les femmes, aux droits humains et au développement, le Programme SAT cherche à trouver de nouvelles et meilleures voies pour tout ce qui concerne la prévention, les recherches et la pratique s'agissant du VIH/sida.

Pour en savoir plus sur le Programme SAT, veuillez contacter Brenda Millar, Agente de programme, Tél. : 613-725-3769, poste 174, courriel: bmillar@cpha.ca

Gerald H. Dafoe

Chef de la direction 